\title{
New Mobile Service Development Process
}

\author{
Hans-Peter Hutter and Andreas Ahlenstorf \\ Zurich University of Applied Sciences (ZHAW), School of Engineering \\ Technikumstr. 9, Winterthur, Switzerland, \\ hans-peter.hutter@zhaw.ch, andreas.ahlenstorf@zhaw.ch
}

\begin{abstract}
Mobile applications play an ever growing role in everybody's life around our globe and the leading app stores currently offer more than 2 million different apps each for their users. It is well accepted that the usage context is much more important in the UI and UX design of these apps than when designing desktop applications. It is important to realize that a lot of these apps are part of a mobile service that defines their usage context and the UX of the mobile app is not only determined by the interaction with it but by the value creation of the whole service. We therefore propose in this paper a joint service and app design process that not only optimizes the user interaction with the mobile app but also the UX of the whole service in order to provide an optimal value proposition to the service customer of the mobile service.
\end{abstract}

Keywords: mobile service, mobile app, service design process

\section{Introduction}

Various user-centered design processes for applications and mobile apps have been proposed in the literature, e.g., [3], [2]. These point out the importance of understanding the user and his needs as well as the usage context. These processes neglect, however, that mobile apps often are part of a mobile service and the usage context and user needs derive from the service needs. On the other hand, different service design processes have been described ,e.g., in [1],[5],[6]. However, none of these processes describe how to derive the UI design requirements for the mobile app from the designed service concept so that the value proposition of the service concept is really achieved.

In this paper a new joint design process is described for mobile apps together with the services they support in order to provide optimal user experience and value proposition to the service customer and app user. First, the basic idea of the new service design process, in the following called InIT Service Design Process (ISDP), is explained.

The second part examplifies how the ISDP can successfully be applied in a real project in the design of a service for the Swiss fair-trade company gebana. The goal of this project was to develop an Access-To-Market (A2M) service for small holder organic farmers in developing countries in order to give them access to a fair-trade market in Europe [4]. This service supports producers of 
organically farmed products to enable them to get access and relationship to a consumer market in the developed countries. It leads them trough the whole process of product tests, organic certification, and setup of the supply chain until a long-term producer-consumer relationship is established. This A2M-service is based on a communication service named MOSAFA. For this service a mobile app had to be developed, that supports the farmers in developing countries to communicate and exchange information with gebana during the whole process.

\section{Basic Idea}

The basic idea of the ISDP is depicted in Figure 1. In a first step the jobs of the targeted customers are identified. Jobs in this context mean e.g. something the customer wants to have done or a feeling he wants to have or to avoid, or an experience he wants to make. Related to these jobs the customers have specific pains and expect specific gains that should be addressed by a service.

For the jobs, gains, and pains that are important to the customer one or more service concepts are designed that have a clear value proposition regarding the jobs of the customer and his pains and gains. These service concepts are iteratively prototyped and evaluated with the customers until promising service concepts are found. A service concept normally addresses only a few jobs, pains or gains of the customer but does this in a convincing manner.

From these service concepts requirements are derived for the mobile app and the other IT systems needed. The mobile app can well support more than one service. The service concepts thereby exactly specify the usage context for the different interaction scenarios of the mobile app.

During the development of the mobile and other systems these are regularly tested in the context of the developed service concepts in order to optimize the user interactions with the app and other IT systems as well as the service concepts themselves.

Customers: Jobs, Gains, Pains

Services

IT-Systems/Apps

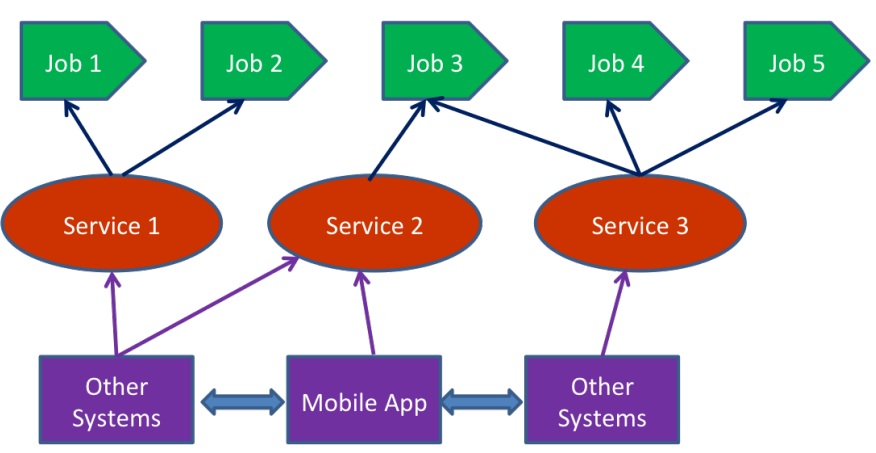

Fig. 1. Basic idea of the ISDP service design process 


\section{Overview on the InIT Service Design Process (ISDP)}

The ISDP is customer centered from the very beginning and iterative by nature (Figure 2):

In the Customer \& Context Analysis phase the service customers and stakeholders are identified and their needs, skills, goals and contexts are investigated, e.g. with contextual interviews, customer journeys and other user research methods (see e.g. [5]). The goal of this phase is to get a clear picture of the service customers and the usage context. The insights of this phase are summarized in service design personas for the different customer groups.

In the second phase, Service Innovation, the first step is to analyze the whole service system with the relevant service customers. Then, the jobs and outcomes of the different service customers are scrutinized in order to identify opportunities for value-creation for each partner in the service system. This can be done with one of the Universal Job Maps described in [3] depending on the innovation focus.

In the Service Design and Prototyping phase the new service concepts for the relevant service customers are iteratively designed addressing their highopportunity jobs, gains, and pains. This done in close collaboration with the targeted service customers in order to optimally support their jobs and to produce the expected outcomes so that the intended values are really created for each service partner. The service concepts are prototyped with value proposition canvases [4] and storyboards in early stages, later on with low-fidelity and high-fidelity prototypes and service-blueprints.

In the evaluation phase, the different service prototypes are evaluated in usability tests and in field tests with real service customers in the real context. The phases Service Design \& Prototyping and Service Evaluation are iterated until the service concept meets the customers' and stakeholders' expectations. If the Service Evaluation phase reveals that additional customer segments have to be addressed for the whole service system the overall service design process is iterated (see Figure 2).

In the Service Implementation phase contextual stories for the personas developed in the Customer \& Context Analysis phase and a domain model are derived from the service blueprints and other user research artifacts. The contextual stories describe the different service provider steps from the customer point of view whereas the domain model describes the universal domain language and, to some extent, the customers mental model. Together with the service prototype they are part of the requirements for the design of the mobile app and the other IT-systems needed to optimally support the designed services. Finally, the mobile app is developed in an iterative user-centered manner like, e.g., described in $[1,2]$. The wireframes and mockups of the mobile app are regularly tested with service customers in the actual service context. 


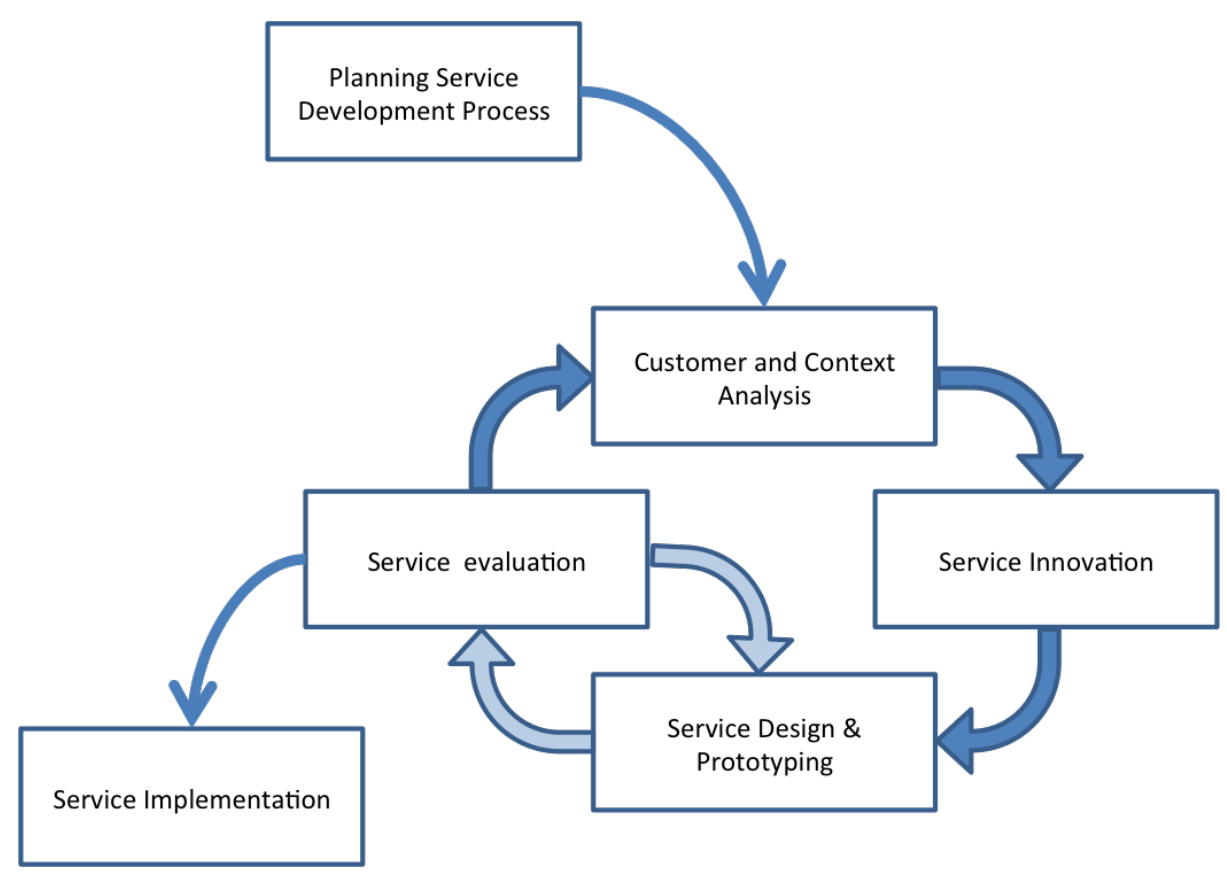

Fig. 2. ISDP overview

\section{Application of the InIT Service Design Process to the MOSAFA service}

In the following it is shown how the different steps of the ISDP looked like in the sample service MOSAFA. The MOSAFA service should allow farmers in developing countries to exchage information with gebana employees in the context of the A2M-service decribed in Section 1.

\subsection{Customer \& Context Analysis}

In the Customer \& Context Analysis phase different contextual inquiries were conducted with farmers in developing countries and the consumers in the developed countries but also with gebana employees in the developing countries and in Switzerland in order to

- Identify the different user groups and other stakeholders important for the MOSAFA service

- Understand the sociocultural and technical context of the users of the MOSAFA service

- Identify the jobs and outcomes (gains and pains) of the different service customers and stakeholders 


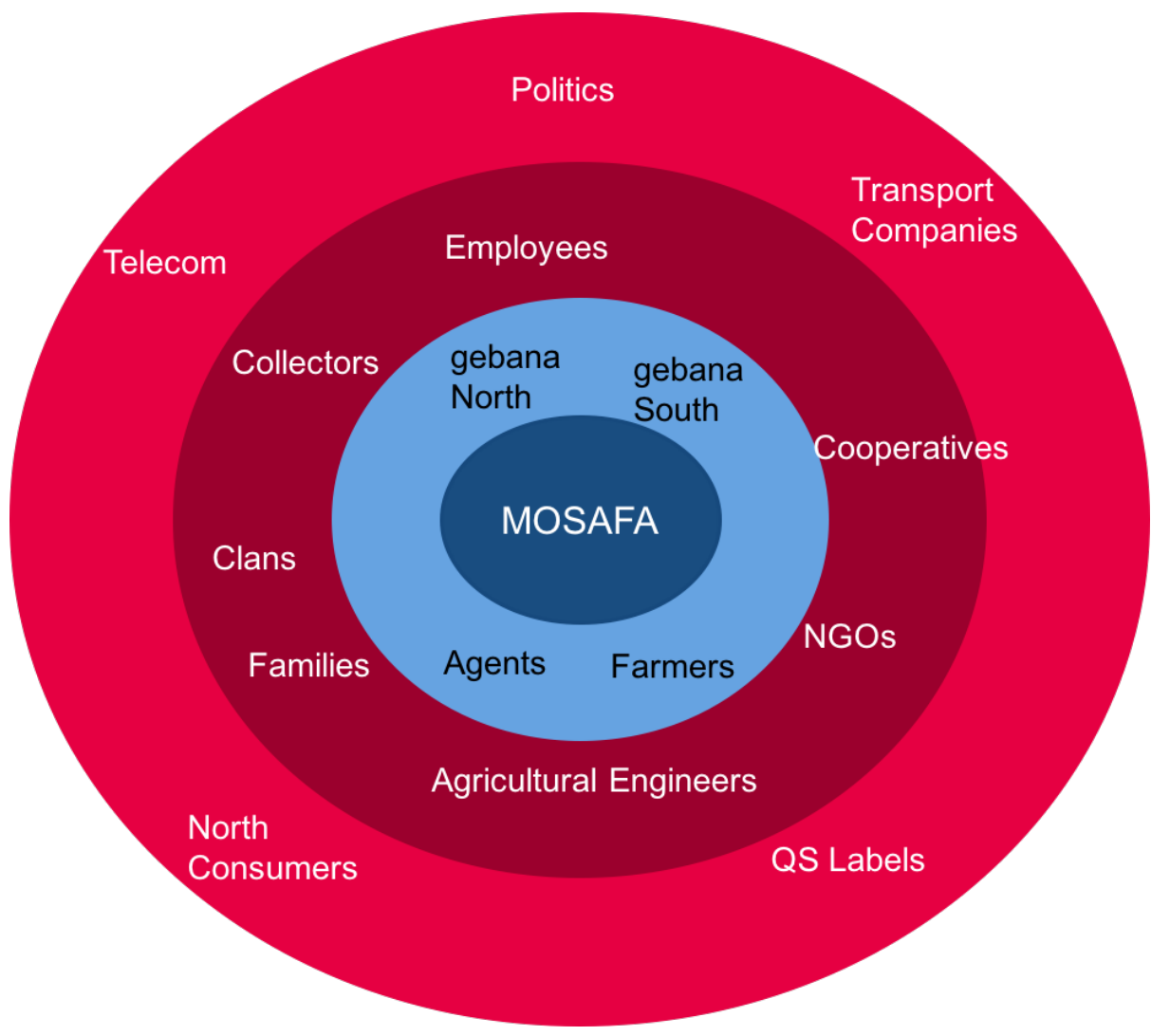

Fig. 3. Stakeholder map for the MOSAFA service

- Elicitate other requirements for the MOSAFA service and app

The stakeholder map in Figure 3 shows the various stakeholders identified during the contextual inquiries. These inquiries revealed that the most important customers of the MOSAFA service are the farmers in developing countries and the gebana management employees in Switzerland and in developing countries. However, the inquiries also revealed significant barriers for the farmers in developing countries to participate in the MOSAFA service:

- Language barriers: the farmers in developing countries often only speak their native language (e.g. Djoula in Burkina Faso) and hardly any major language like English or French. This problem may be alleviated in the future, as the kids of the farmers normally learn some English or French at school.

- Technical barriers: although most of the farmers have, or at least have access to, a mobile phone, they normally only use it for phone calls. Several of the interviewed farmers have no experience with the internet nor with mobile apps. 
Therefore, another customer group was introduced for the MOSAFA service: the agents. Whereas the gebana employees interact directly with the MOSAFA service the farmers in the south normally do not directly interact with the MOSAFA app but through an intermediate, the so-called agent. An agent is responsible for a group of farmers that he regularly contacts and communicates with. The role of an agent may be taken over by a representative of the farmer cooperatives, a Collecteur (see below) or someone else who has good contacts to the farmers and is knowledgable in English or French and has some basic IT skills.

Other stakeholders of the MOSAFA and the A2M service worth mentioning here are:

- Collecteurs: these are persons that collect the harvested goods from the farmers and transport them to a processing station, e.g., a drying station, or a storehouse.

- Agricultural Engineers: these are persons paid by gebana in order to advise the farmers on organic farming.

- NGOs: they also have direct interest in the A2M-service in two ways: On the one side they are looking for market access for their farmer groups in the developing countries, on the other side they are looking for fair-trade projects in developing countries they could support.

During the Customer \& Context Analysis phase also the technical context for a communication service like MOSAFA was analyzed. The major insights and conclusions of the analysis were:

- Messaging Services

- Data connection was normally stable enough to transmit messages. However, none of the Messaging Services tested (SMS, Pushy, Google Cloud Messaging) was reliable enough to transmit information without any losses.

- There were very long delays (up to several days) in the data transmission and no guarantee for any upper limit.

- Data connection

- The quality of the data connection was quite variable even for the same connection

- Often there is no data connection at all for longer periods of time (days)

- Outside gebana offices there was no WiFi connection available

- Breakdown rate during data transfers were high. Only $20 \%$ of the data transmissions (10 MB) were completed.

- On average only 0.5 MB data could be transferred in one transmission.

- Downstream: 10-100 MB per day could be transmitted

- Upstream: only up to $5 \mathrm{MB}$ per week could be transmitted

These insights lead to the following conclusion regarding the MOSAFA service and the corresponding mobile app:

- It is generally possible to exchange information digitally. 
- Reliable exchange of digital information with partners in developing countries is a challenge.

- Information exchange with partners in developing countries via SMS or pushnotifications is not reliable enough.

- The best solution therefore seems to be the following:

- The applications involved in the data exchange must be resilient to frequent connection losses and should support partial data transmissions without the need for retransmissions.

- Information exchange is therefore best done via a generic form app to be developed for smartphones. With this app partners in developing countries can collect the data directly on the mobile device on site. The data is first stored on the mobile device. As soon as an internet connection is available the data is automatically transferred to a server at gebana.

- A web server at gebana offers a web service that can be called from the mobile app in the global south as many times as needed (pull service).

- With this concept it should be possible to reliably collect data via a form app in reasonable time. However, there is no guarantee that time-critical information can be transmitted in time.

- Large contents such as pictures or videos should only be transferred when a reliable WiFi connection is available, e.g., at the gebana offices.

- All data transfers should be done automatically in the background (e.g., via the SyncAdapter in Android) in order to save battery on the mobile and to automatically transfer data as soon as a network connection is available.

Based on the results of the contextual inquiries the following three most important service customer groups of the MOSAFA service were indentified: farmers, agents, and gebana employees. For each of these service customer groups a service design persona was developed.

\subsection{Service Innovation}

In this phase the jobs, gains, and pains of the three personas developed in the last phase are scrutinized. It is important to realize that the MOSAFA service relies on three service customers, as depicted in Figure 4. For each of this service customers an attractive value proposition has to be offered by the service so that these customers really participate in the service on a long-term basis.

The farmer provides information to the MOSAFA service needed by the gebana employee. The value he expects from the MOSAFA service is contacts to other farmers, agents, and gebana employees, information regarding organicfarming and advice. The motivation of an agent to participate is - apart from money, contacts to farmers, and information - that he gets a smartphone and an influential role among the farmers. The gebana employee provides tasks for the agents and information to the MOSAFA service. The value he gets out of it is an easy way to run data collection campaign and collect data from farmers and agents. 


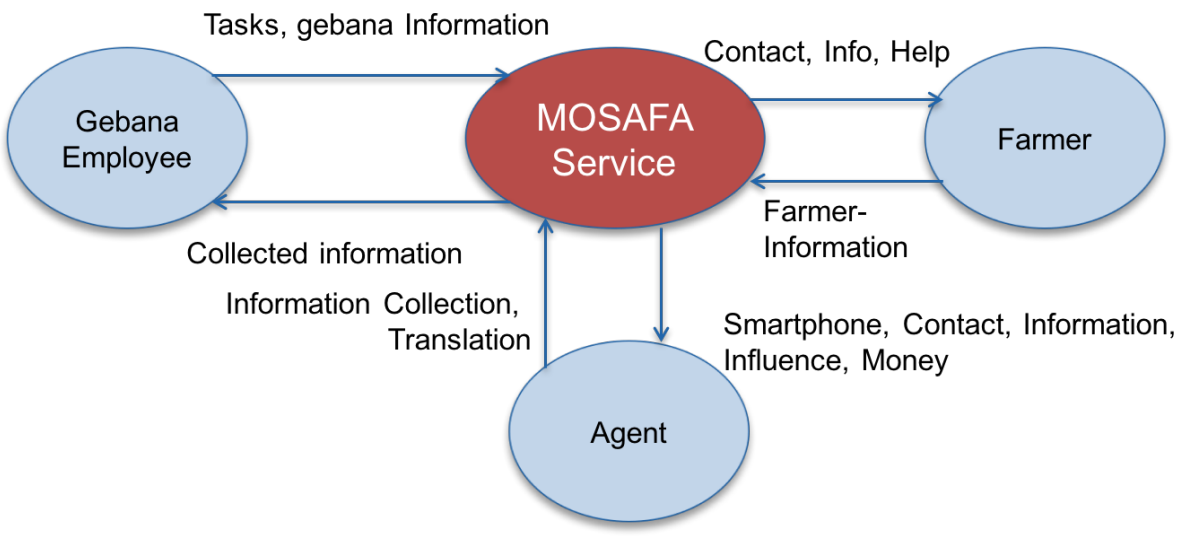

Fig. 4. Service system of MOSAFA

For each of these three service customers the different jobs they want to have done were identified together with the gains and pains related to these jobs. The prioritizing of the gathered list of jobs and outcomes revealed the following jobs, gain, and pains that should primarily be addressed by the MOSAFA service:

- Gebana employee

- Jobs

* Run data collection campaign

* Collect farmer profiles

* Collect agent profiles

* Collect farmers' stock status

* Collect foto/videos from farmers

* Distribute information to farmers

* Assign tasks to farmers

* Assign tasks to agents

* Remind agents

- Pains

* Effort to collect information

* Time to collect information

* Wrong information

* Missing information

- Gains

* Easy verification of collection status

* Flexible aggregation of the data

* Easy visualization of data

- Agent

- Jobs

* Collect farmer contact info 
* Collect farmer profiles

* Collect farmers stock status

* Distribute information to farmers

* Collect other information from farmers (organic certification)

* Verify/Update information on site

- Pains

* Effort to collect information

* Time to collect information

* Information gets lost

* Forget something to do

- Gains

* Easy overview on open/closed tasks

$*$ Be perceived as reliable

* Be perceived as trustful

* Be influencial

- Farmer

- Jobs

* Provide information to gebana

* Receive instructions

* Do organic farming

* Sell products

- Pains

* Farming problems

* Organic certification fails

* No contact to agent

* Low harvest

* Low income

* No market for product

* Cannot sell whole stock

- Gains

* Help with organic certification

* Good price for products

* Help with organic farming

* Exchange with other farmers

* Regular contact to agent

* Contact to gebana

* Access to market in developed countries

In the following the job analysis focused on the core job of the gebana employee Run a data collection campaign together with the related jobs of the agents, and farmers. For this we analyzed the job steps of the gebana employee, the agent, and the farmer involved in this core job using the Universal Job Map proposed by Bettencourt[1]. For each of these jobs steps identified we again looked for the major pains and gains of the personas related to each job step.

From this job map of the core job Run a data collection campaign we also derived a service blueprint showing the most important steps of each of the service partners as well as the interactions between the personas an the MOSAFA app and MOSAFA server, resp. (see Figure 5). 


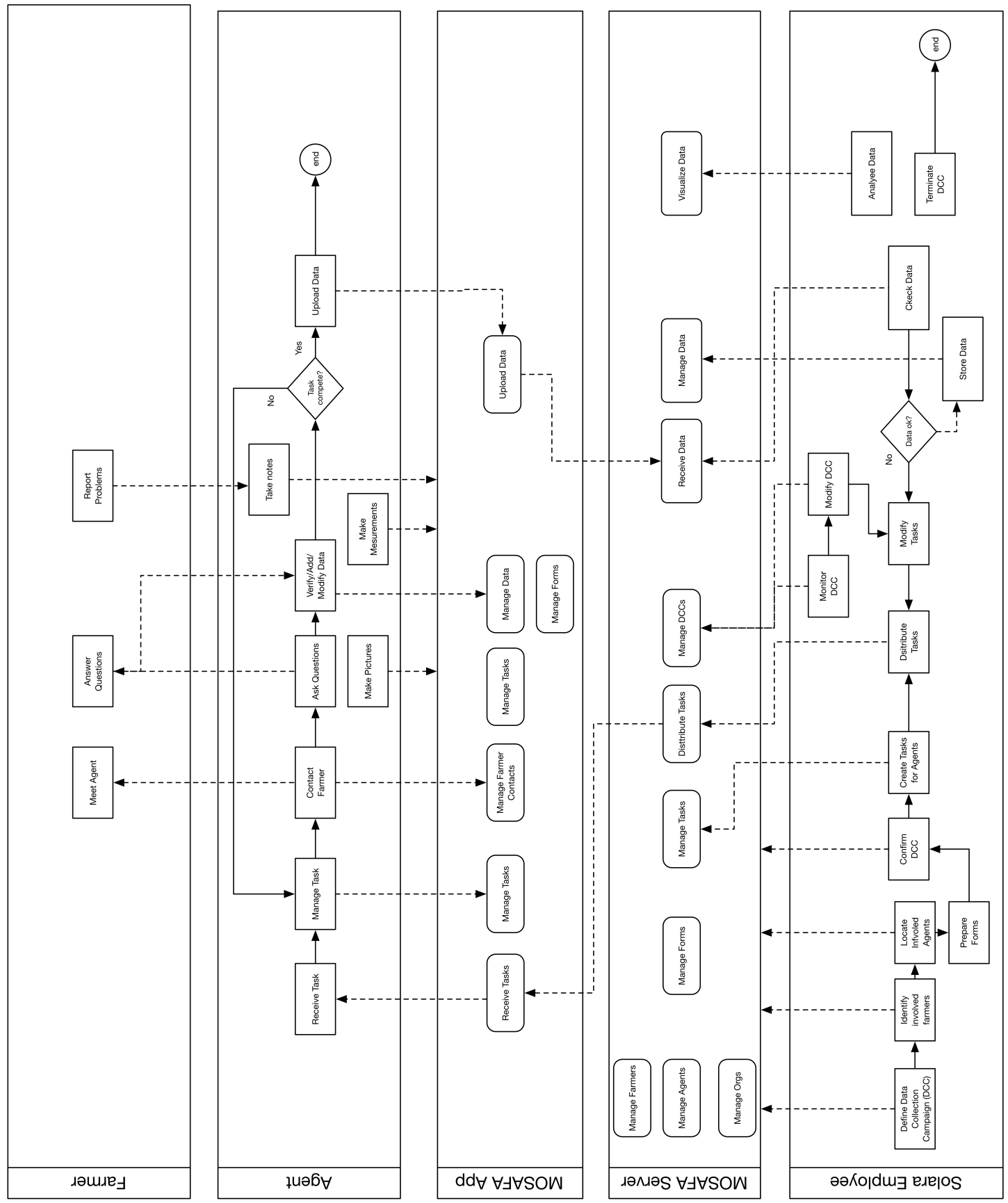

Fig. 5. Service blueprint for the MOSAFA service 


\subsection{Service Design \& Prototyping and Service Evaluation}

From the service blueprint in Figure 5 contextual scenarios were developed for each interaction with the MOSAFA app and the MOSAFA server in the blueprint. In addition, a domain model was developed that shows all important concepts and their relations of the MOSAFA service form the user point of view (see Figure 6). Based on these contextual scenarios and the domain model different mockups of the MOSAFA App and MOSAFA Server were iteratively developed. The mockups were evaluated with usability tests with real service customers in the context of the MOSAFA service.

\subsection{Service Implementation}

The service implementation of the MOSAFA service mainly comprised the development of the MOSAFA app and the MOSAFA server. The requirements for the app were derived from the contextual scenarios and the domain model of the service design phase. The contextual scenarios thereby describe the interaction dynamics where the domain model shows the static view the of the problem domain. Both are valuable inputs for the software development of the MOSAFA app and server in addition to the UI-mockups developed in the service design phase.

The resulting MOSAFA app is a generic form app for the agents that connects to the MOSAFA server as soon as an internet connection is available and automatically downloads the tasks for the agent. A task comprises a description and a form for each farmer that is part of the data collection campaign. The agent can easily enter the data into the form while talking to the farmer. He can also take additional notes and pictures right in the form. The data entered is stored locally on the mobile device. As soon as the mobile device has internet connection the filled forms are uploaded to the MOSAFA server. Pictures are only uploaded in there is a broadband connection, e.g. W to the MOSAFA server. Such a task can also be used to distribute information to the farmers or agents. The MOSAFA app gives the agent an easy overview of all his tasks and their status.

On the MOSAFA server the gebana employee can easily set up a data collection campaign selecting the corresponding farmers and agents. He then defines the task for the agents and selects or creates the corresponding form type. Based on this information the MOSAFA server automatically generates all individual tasks and forms for the different agents. Once the data collection campaign has started, the gebana employee has an easy overview on the status of the data collection campaign, i.e., which tasks are pending, which are completed, which forms have been returned, and so on.

\section{Conclusion}

This paper proposes the first time an overall design process model for a mobile app as a part of a mobile service which covers the whole process from the analysis 
of the service customers, their context, jobs, gains and pains, through the service design down to the interaction design and the development of the corresponding mobile app. This allows that the UX of the mobile app and the value proposition of the corresponding service are optimized in a joint effort.

\section{Acknowledgement}

This work was supported by the Consortium for Technology and Innovation CTI of Switzerland in the project CTI 16331.1 PFES-ES.

\section{References}

1. Bettencourt, L.A.: Service Innovation. How to Go from Customer Needs to Breakthrough Services. Mc Graw Hill, New York (2010)

2. DIN/EN/ISO: International Standard DIN EN ISO 2941-210:2010. Ergonomics of human-system interaction - Part 210: Human-centred design for interactive systems. International Organization for Standardization, International Electrotechnical Commission, Geneva (2010)

3. Direkova, N.: Design sprint methods (2016), https://developers.google.com/ design-sprint/downloads/DesignSprintMethods.pdf

4. Hutter, H.P., Klammer, J., den Anker, F.V., Wiedmer, A.: Service platform for the exchance of services with developing countries. In: Tech4Dev 2016, UNESCO Chair in Technologies for Development Int. Conference, Lausanne. p. 127 (2016)

5. Osterwalder, A., et al., Y.P.: Value Proposition Design: How to Create Products and Services Customers Want. Strategyzer Series, John Wiley \& Sons Inc., New Jersey (2014)

6. Stickdorn, M., Schneider, J.: This Is Service Design Thinking. Basics - Tools Cases. BIS Publishers, Amsterdam (2011) 


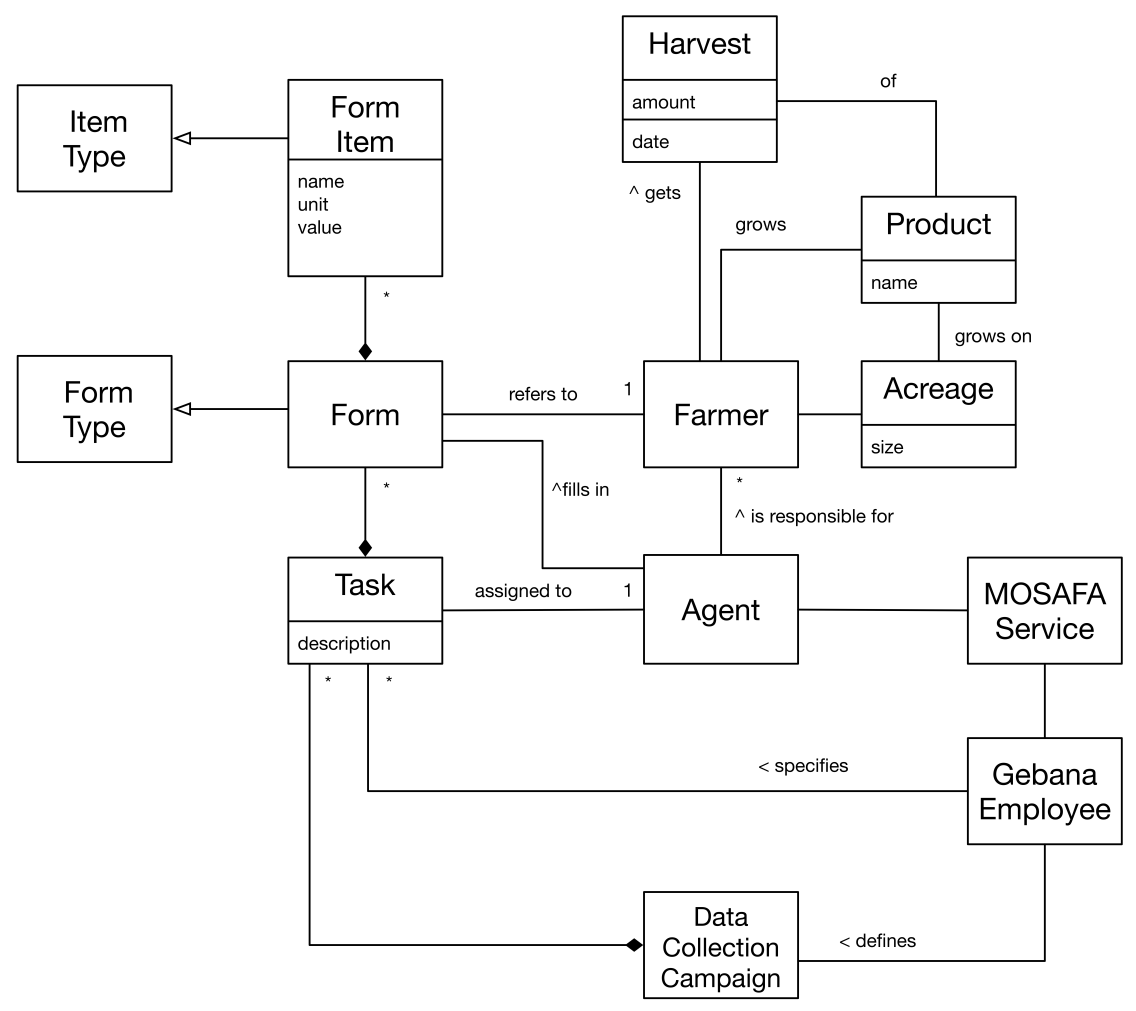

Fig. 6. Domain model for the MOSAFA service 